\title{
Modeling the Structure of Web Applications with ArgoUWE
}

\author{
Alexander Knapp, Nora Koch, and Gefei Zhang \\ Ludwig-Maximilians-Universität München \\ \{knapp, kochn, zhangg\}@pst . ifi.lmu .de
}

\begin{abstract}
The UWE methodology provides a systematic, UML-based approach for the development of Web applications. The CASE tool ArgoUWE supports the design phase of the UWE development process. It is implemented as a plugin module of the open source ArgoUML modeling tool. The construction process of Web applications is supported by incorporating the semi-automatic UWE development steps as well as the OCL well-formedness rules of the UWE metamodel.
\end{abstract}

The increasing complexity of Web applications requires mainly process and tool support. Such support is offered by the UWE ("UML-based Web Engineering" [3]) method and the ArgoUWE [2] CASE too 1]. The main features of ArgoUWE are the support of visual modeling, UML compliance, and open source characteristic. The design of a Web application is performed according to the UML-based notations and the steps defined by the UWE method.

UWE is an UML-based, object-oriented, iterative, and incremental approach for the development of Web applications. In a single iteration, a use case model, a conceptual model, a navigational model, and a presentation model are built or refined. The use case model reflects the requirements on the Web application and the conceptual model comprises the entities the Web applications is based on. The navigational model builds on the conceptual model, presenting the navigation structure of the Web application between the different entities and offering means for accessing the entities through querying, menu selection, etc. The presentation model refines the navigational model by adding the layout. The presentation model complements the navigation model by giving the layout view. All these models of a Web application are supported by convenient UWE notations that are built on UML notations. The UWE process defines the development steps for building Web applications and, in particular, includes semi-automatic tasks for generating the navigational model from the conceptual model and the presentation model from the navigational model.

ArgoUWE supports the structural modeling tasks of UWE. On the one hand it offers tailored editors for the UWE notations used for conceptual, navigational, and presentation modeling of Web applications. On the other hand, ArgoUWE provides several semi-automatic model transformations that occur in the UWE process. As these model transformations are UWE-metamodel-based, both consistency between the different models and integrity of the overall Web application model with respect to UWE's OCL constraints are ensured by the tool. In particular, in a conceptual model classes can

\footnotetext{
${ }^{1}$ http://www.pst.ifi.lmu.de/projekte/argouwe
} 


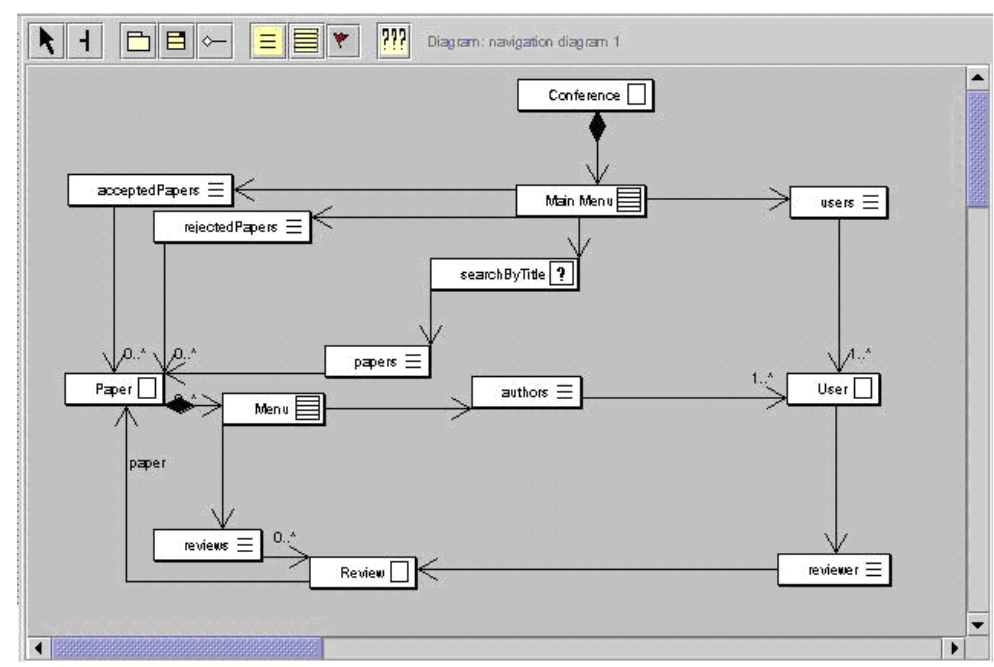

be marked for navigation and the annotated conceptual model can be turned into a navigational model by creating navigation classes and associations for marked conceptual classes. In the navigational model, ArgoUWE can add automatically access primitives, like queries and menus, between navigation classes that refine the navigational structure (see figure above). Finally, ArgoUWE can infer a presentation model from a navigation model automatically. Consistency of models is not enforced during modeling, but can be triggered any time by the user thus supporting and not constraining the developer in creating models. For a comparison with other tools we refer to [2].

ArgoUWE is implemented as a plugin into the open-source UML modeling tool ArgoUML [1]. ArgoUWE reuses the UML-metamodel-based modeling techniques of ArgoUML. The support of ArgoUML for UML's standard model interchange format XMI is put to use by an extension of XMI by the Web modeling profile of UWE. ArgoUML being an open-source tool with an active developer community, plugin developers have to face rapid version changes and a sometimes poor documentation. However, the interest of availability and the possibility to adapt ArgoUWE, which again is open-source, to user-specific needs outweigh these disadvantages.

ArgoUWE is part of the OpenUWE tool environment for model-driven generation of Web applications. ArgoUWE is used in design whereas UWEXML is employed for semi-automatic generation of Web applications from the design model.

\section{References}

1. http://www.argouml.org.

2. A. Knapp, N. Koch, F. Moser, and G. Zhang. ArgoUWE: A CASE Tool for Web Applications. In Proc. $1^{\text {st }}$ Int. Wsh. Engineering Methods to Support Information Systems Evolution (EMSISE'03), Genève, 2003. 14 pages.

3. N. Koch and A. Kraus. The Expressive Power of UML-based Web Engineering. In D. Schwabe, O. Pastor, G. Rossi, and L. Olsina, editors, Proc. $2^{\text {nd }}$ Int. Wsh. Web-Oriented Software Technology (IWWOST'02). CYTED, 2002. 